\title{
Method of Designing a Software Analytical Complex Based on a Cartesian Closed Category Using Virtual Objects
}

\author{
Gennady Kulikov \\ Faculty of Informatics of Robotics \\ Department of automated control and \\ management systems \\ Ufa State Aviation Technical \\ University \\ Ufa, Russia \\ gennadyg_98@yahoo.com \\ Lyudmila Rodionova \\ Faculty of Informatics of Robotics \\ Department of automated control and \\ management systems \\ Ufa State Aviation Technical University \\ Ufa, Russia \\ lurik@mail.ru
}

\author{
Vyacheslav Antonov \\ Faculty of Informatics of Robotics \\ Department of automated control and \\ management systems \\ Ufa State Aviation Technical University \\ Ufa, Russia \\ antonov.V@bashkortostan.ru
}

\author{
Maria Shilina \\ Faculty of Informatics of Robotics \\ Department of automated control and \\ management systems \\ Ufa State Aviation Technical University \\ Ufa, Russia \\ maria.shilina@gmail.com
}

\begin{abstract}
The article examines the problem of formalization and identification of information objects (real and virtual) and traceability of their relations in the studied subject area according to the rules of monomorphism and polymorphism of the mathematical theory of categories of sets. Based on these conditions, a formal model of interaction processes of the components of a software analytical complex with a Cartesian closed category and a set-theoretic method for mapping the functional interaction of software systems in accordance with the principles of ISO 15288 are developed. It is shown that the structure of such software analysis complex satisfies the conditions of Cartesian closed logic, which significantly increases the range of tasks. This is achieved by possibly conducting effective software reengineering without changing its base code in real time. A formalized description of the relationship between virtual and real objects in symbolic form according to the rules of the data warehouse is proposed. The method of constructing hierarchies by N. Chomsky and the method of semantic differentials by $\mathrm{C}$. Osgood are applied. As an example, the structure of a distributed software analytical complex for managing the personnel reserve of an industrial enterprise, which is being developed jointly with educational institutions of higher education, has been modified.
\end{abstract}

Keywords-Cartesian closed category; virtual and real objects; formal model; subject area; identifiability and traceability.Introduction

\section{INTRODUCTION}

The development of science and technology. The globalization of the economy, integration processes in civil society cause the need to create more advanced defense, educational, industrial, transport, energy and other systems. In response to the requirements of development, these systems are constantly becoming more complex: in the composition of which more and more elements appear, the boundaries become mobile, models that are more difficult for quick perception are used to describe behavior.
The use of systems engineering standards allows us to describe the concepts and management of activities to create systems of any scale and purpose. Today, special attention is paid to software analytical complexes with elements of intelligent control that can not only generate regulatory information and reports, but also provide an opportunity for in-depth analytical work by decision makers. There is an interest in the use of big data analytics, the categories of information objects - software modules that provide the implementation of the connection of real and virtual objects with information processes, taking into account their mutual relations in the software analytical complex (SAC). All of this can also be added to Big Data problems.

Thus, the AOS Big Data can be represented different categories of objects, linked by relations, there is the problem of categorization and clustering. Thus, it becomes relevant the design and development of software analytical complex (SAC), which can be used by large companies, tasks of personnel management, personnel reserve, which is increasingly determined by efficiency criteria. The next problem of the study is the formalisation and identification of information objects and maintaining their compliance with the real objects of the researched subject area, as well as compliance with traceability of information and the actual settings objects for the knowledge base SAC. To define real objects, matching them to information objects and traceability at the abstract level of the studied educational production systems applies concepts and rules of ISO/IEC/IEEE 15288.

\section{THE RELEVANCE OF RESEARCH}

So, for the last 5 years the volume of stored information has increased 100 times, the Amount of data around the world in 2025 will increase by 10 times. 
Accordingly increases the amount of data and information in electronic form. An increasing number of automated systems related to the management of manufacturing resources (ERP system). Therefore, the control objective in this case is faced with the problem of handling heterogeneous data. And also there is an increase in the number of written software systems, but the disadvantage of these software products is that they are not fully developed for specific processes of the enterprise. As a result, for a particular subject area necessary to produce the categorization, abstraction and unification of these systems in software systems. In order to fully provide the necessary information.

Thus, there are many management tasks, many software systems, there is the problem of choice of software tools and capabilities integrated use of these software tools, through the use of analytical systems. Formalization of control tasks subject area and transfer them into appropriate software analytical systems provide information according to

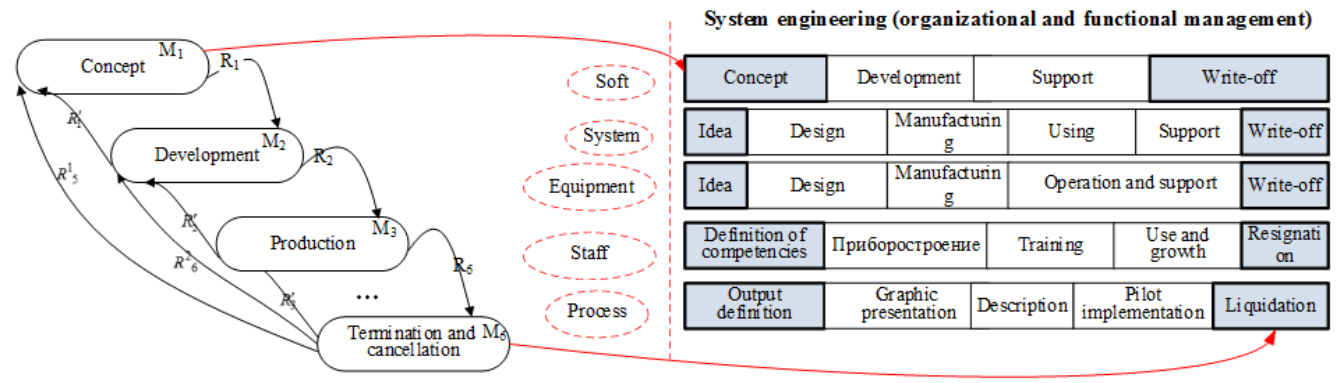

General modeling theor

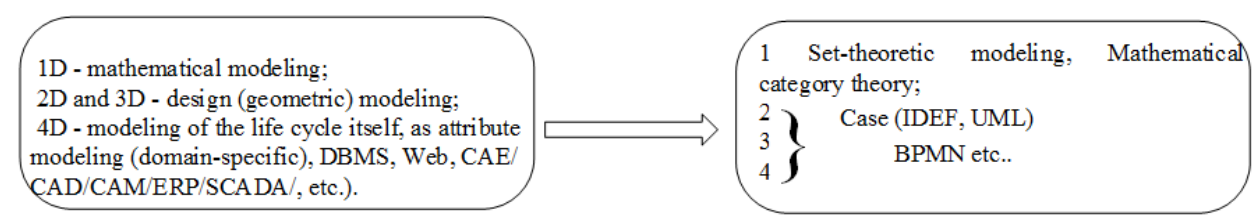

Fig. 1. Compliance modeling methods used in engineering practice, systems engineering

III. APPLICATION OF THE SYSTEM ENGINEERING STANDARD FOR THE INITIAL FORMATION OF A DOMAIN MODEL

management action.

In General, you need to integrate in terms of semantic heterogeneity, incompleteness and inaccuracies of the data.

The use of set theory and category theory is considered as one of the ways associated with the formalization of these approaches.

It is proposed to use methods of category theory, standards, system engineering ISO15288.

It technologies offer new opportunities, focusing on that there is a change (adjustment) regulatory framework - reengineering AT BP. As a consequence of the growing complexity of the structure of the PC, delay in time of their design and re-engineering. All this is happening against the background of increasing interest with public services in electronic form. This slide shows the quantitative characteristics and qualitative characteristics of this.

There is a task of unified life cycle life cycles of software, IP, PC

The number of re-engineering processes of state regulation during this time increased by $80 \%$, project development SMEV - 12 times. Growth of PC is accompanied by a lack of return on funding (mostly reverse engineering), and the inexpediency of increase of it professionals. All this is accompanied by intensity the number of running state services. Given the specificity of this information and broad interaction with society, it is necessary to resolve the issue of traceability and the possibility of repetition of any previously issued information.
The principles of systems engineering are based primarily on the construction of formalized or formal models of life cycles (LC) of the system under study and its elements in various aspects. In this sense, we define the main stages of life cycle - the development and application of methods for digital modeling of production processes as systems (Fig. 1.).

Today, there is engineering activity for domain objects, which, from the point of view of modeling theory, is classified as follows:

1D - this is math (design tools, for example, Mathematica software for mathematical calculations of physical processes ANSYS);

2D, 3D - This "spatial topology (diagrams, drawings, etc., is amenable to formalization, either flat or volumetric, CAD, CAD, etc.)" [1];

Standards of system engineering for the design of organizational-functional systems, such as (ISO / IEC / IEEE 15288: 2015, ISO / IEC / IEEE 42010: 2011, OMG Essence and others), which also have analogues 1D, 2D (functional model), 3D (many functional models), 4D (PDM / PLM systems, engineering methodology).

In the standard of system engineering, which define objects as systems (software systems, and system personnel, system equipment, process systems). This standard provides the functional and organizational management of the life cycle to the objects of the system. Vertically structured display of objects. 
We get two measurements along the vertical - structural mapping (static), and horizontally - the sequence of actions (dynamic).

In the particular case, consider the example of the formation of personnel reserve of the enterprise. Providing data of personnel reserve specialists allows the enterprise not to be in shortage of personnel in case of dismissal, transfer.

The tool of improvement for the formation of personnel reserve is intellectual content, which will allow to formalize the data of the specialists who form this reserve [2 -4].The abbreviation "i.e." means "that is", and the abbreviation "e.g." means "for example".

Content is a filled information space in various ways (text, pictures, audio, video, etc.) $[5,6]$.

Intellectual content is determined by a set of information (real and virtual), data and knowledge, their relationship parameters, preserving knowledge about the subject area.

Human capital is the amount of accumulated knowledge and skills, motivations and energy that can be used for a certain time to produce goods and services [7].

Filling intellectual content will allow you to use structured data and knowledge for the formation of personnel reserve of the enterprise.

The personnel reserve is a group of specialists, potentially aspiring to a managerial position, who have passed the competitive selection and individual training.

According to many authors, there are several categories of personnel reserve, it all depends on the objectives of the personnel policy of the enterprise. The objectives of personnel policy can be: the ability to select personnel for certain positions, saving and growth of the personnel potential of an enterprise, recruiting highly efficient managerial personnel capable of realizing the functions and tasks of an enterprise $[8,9]$.

The reserve is clustered by type of activity on the development reserve / strategic reserve (groups of data on specialists ready to work in new conditions, for example, when diversifying production) and the reserve of functioning / operational reserve (groups of data on specialists ready to work on managerial (management) positions). The reserve can also be classified according to the time of appointment: Group A - data of specialists who can start work in this position in the near future, and Group B - data of specialists who are included in job planning for 2-3 years [10 - 12].

The tasks of personnel reserve: the implementation of staff rotation, training candidates for unforeseen cases of staff retirement, planning career paths.

\section{THE SET-THEORETIC METHOD FOR MODELING PROCESSES FOR DESIGNING A SOFTWARE ANALYTICAL COMPLEX}

To build a logically consistent structure SAC, the system engineering standard ISO 15288 was applied, considered on the basis of one agreement process, which was decomposed into two: the process of acquisition and supply, according to the requirements of system engineering. Thus, each stage can be decomposed to a certain level. The agreements that make up the process, the acquisition process and the delivery process (properties) are complex objects, so we decompose them into sub-objects: process goals, activities and results in accordance with the requirements of systems engineering. Relations between them are defined on the basis of functor relations. In general, a chain of relationships is maintained, which can be passed from the top to any end point. No matter the process of purchase or delivery. It turns out the multiplicity of chains. In general, the number of chains may be small. In accordance with the requirement of the required Ashby variety, the number of relationships must be at least two for each object. The interaction of these objects opens up the possibility of further composition of their objects in the form of completion. New relationships allow you to finish building to Descartes square. If two objects do not interact with each other, then the system is not fully examined.

In a real system, there are a certain number of functions. Each chain can be completed to Descartes square, as a result of which a recurrent path appears. And as long as the system retains its integrity, it will be unique. The problem of traceability of all system objects is solved, and relations can also act as objects. As a result, there are more Requirements identifiability and traceability at all stages of the creation and operation of the SAC.

Obviously, with the decomposition of the system into subsystems (selection of new objects and connections), the number of partially overlapped areas (the so-called redundancy) increases. The size of the formalized part is determined by the sum consisting of the number of objects and the number of links.

Thus, the problem of applying formal methods of process modeling for the design of a SAC can be solved by applying the mathematical apparatus of category theory using the ISO/IEC/IEEE 15288 standard and the information approach of A. A. Denisov.

We formalize the considered conditions in the form of a matrix (an analogue of J. Zahman's matrix), where the columns represent the phases of the life cycle of a PAK, and the lines represent business processes that ensure the indicated stages according to ISO / IEC / IEEE 15288 (Fig. 2).

We introduce the following notation:

$$
\text { 1. Life cycle stages }-M=\left\{M_{1}, \ldots, M_{6}\right\} \text {. }
$$

2. Multiple agreement processes -

$$
P S=\left\{P S^{M 1}, \ldots, P S^{M 2}\right\} \text {. }
$$

3. Many project processes -

$$
P P R=\left\{P P R^{M 1}, \ldots, P P R^{M 2}\right\} .
$$

4. Many enterprise processes -

$$
P P=\left\{P P^{M 1}, \ldots, P P^{M 2}\right\} \text {. }
$$

5. Many technical processes -

$$
T P=\left\{T P^{M 1}, \ldots, T P^{M 2}\right\} \text {. }
$$

As a result, we obtain a multidimensional table that defines the architecture of the structure of the semantic aspect of the information system model and the software 
analytical complex that implements it, taking into account the interaction of functional programs with the environment.

The model allows you to create both the structure of the life cycle of a SAC in the life cycle space of a business process, and the structure of the life cycle of individual BPs within the framework of the project being implemented.

The software analytical complex is built on the same relationships. Those. each object of the system is the module SAC, which is determined by the ratio. The information set of objects generated by this method determines the content. Built on this method, the ACL is completely determined by a set of accounting objects, including modules of relations between them with the maximum transfer of semantics into syntax and writing to objects.

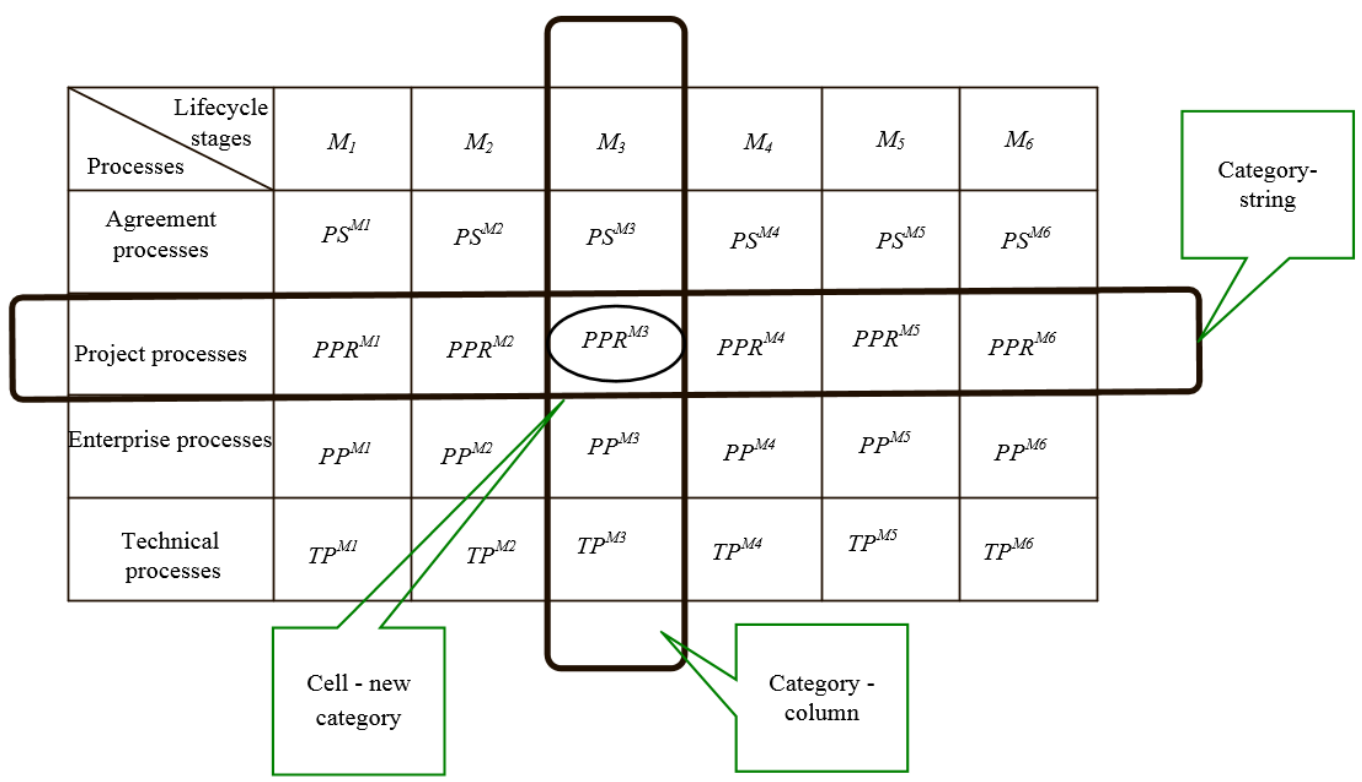

Fig. 2. The architecture of the semantic model of the enterprise activity system

In the next step, assuming that having many structures, we can define one of the consistent data warehouse structures for the personnel reserve management agreement process model [13, 14]. Based on the model, we can determine the SAC scheme (Fig. 3). Obviously, the SAC structure meets the same architectural requirements. SAC is characterized by the following parameters: using various database management systems (Oracle, MySQL, etc.), modern storage facilities (DataWarehouse), application of online analytical processing (OLTP - Online Transaction Processing, OLAP - On-Line Analytical Processing), intellectual analysis tools data (data mining), etc.

As a result of using SAC, users are decision makers (top and middle managers, as well as analysts) get an effective tool for selecting, analyzing candidates for personnel reserve, as well as for generating data on personnel reserve, presented in tabular and graphical form. This PAC solves the following tasks (numerous data from candidates for personnel reserve):

1. Ensuring centralized collection and storage.

2. Processing, updating and structuring.

3. Integration and consolidated analysis.

In the PAC subsystems correspond to the principles:

- developability;

- block construction;

- a certain independence of interacting subsystems and their databases (DB);

- adaptability of the functional part, etc.
All SAC objects presented in figure 3 can be defined as separate categories with relationships among themselves, according to the previously described method.

For the particular case of personnel reserve management, the main data for the database is the candidate data directory, where there is complete information about the candidate, the identifier of the table in which the attribute values are stored, and also:

1. The identifier of the group to which the attribute belongs.

2. The identifier of the dimension of the attribute values.

3. The frequency of the presentation of attribute values.

4. The field of additional information - comments for the attribute.

The knowledge base contains the rules of the algorithm of the program described by the categories of sets [15-17].

The external data sources are the authorities.

state authorities (Ministry of Labor and Social Protection of Population, local administration of the city and region, etc.), interacting organizations (employment centers, universities, colleges, enterprises, etc.), higher management bodies (holdings, corporations, etc.).

The use of a formal description of information objects in the language of category theory makes it possible to increase the formalization of the domain model [18, 19], which opens up new possibilities for identifying problems in the early stages of the development of a SAC. When forming a data warehouse, individual objects expressed by categories of users, categories of program modules can be 
considered, the relations between them will be determined by functors. This allows you to use them in the form of similar accounting objects. Thus, the problem of separation of data and knowledge during processing and transmission is solved.

When building a structure SAC was polykovichskoe data model consisting of many cubes. For example, consider one of them, which consists of: category, user, time and function. One of the functions SAC "working with databases", in turn, are decomposed into types of Database actions: search the database, module database and place the backup database. Thus, all other objects have the opportunity to further decomposition.

The result is a matrix of work with a SAC that defines the characteristics of the used module, depending on time or function. This process is accompanied by inside SAC.

Thus, the proposed description in the language of category theory and fuzzy sets allows to obtain hierarchical information system with full data definition and analytical software system as a complete functioning unit. The developed method allows to form boundaries between the semantic and syntactic description of processes and functional software SAC [20].

As an example, designed a prototype software-analytical complex, considered in a particular case in one of the examples of management processes of formation and maintenance of a personnel reserve of the enterprise, consisting of complex 1C, complex Prognoz Platform and program data interaction of software systems.
The structure of the core intellectual content represents the totality of information-analytical systems with corresponding applications, databases, knowledge bases, etc., it is open, because it is constantly replenished with new structurally-related content management systems. The system allows the use of different modules. This model is built according to the ontological principle. As a tool for building dashboard is used Prognoz Platform 8. This software allows to develop native applications for the formation of analytical documents. On the dashboard shows the performance of personnel reserve: dynamics of the number of candidates for a certain period, the distribution of the age composition, the number of candidates entered into the personnel reserve, the number of candidates eliminated from the candidate pool. Efficiency with no purchasing new modules, and by building additional configurations.

The structure of the core analytical software complex is a set of software systems with corresponding applications, databases, knowledge bases, etc., it is open, because it is constantly replenished with new structurally-related content management systems.

The article content is regarded as information provision one-way description, software, and computing environment. Means of information processing are software tools that can be transferred via communication channels, therefore, they can also be considered content.

The system allows the use of different modules that another step to increase the level of abstraction. Some of the information that was in the semantics into the syntax.

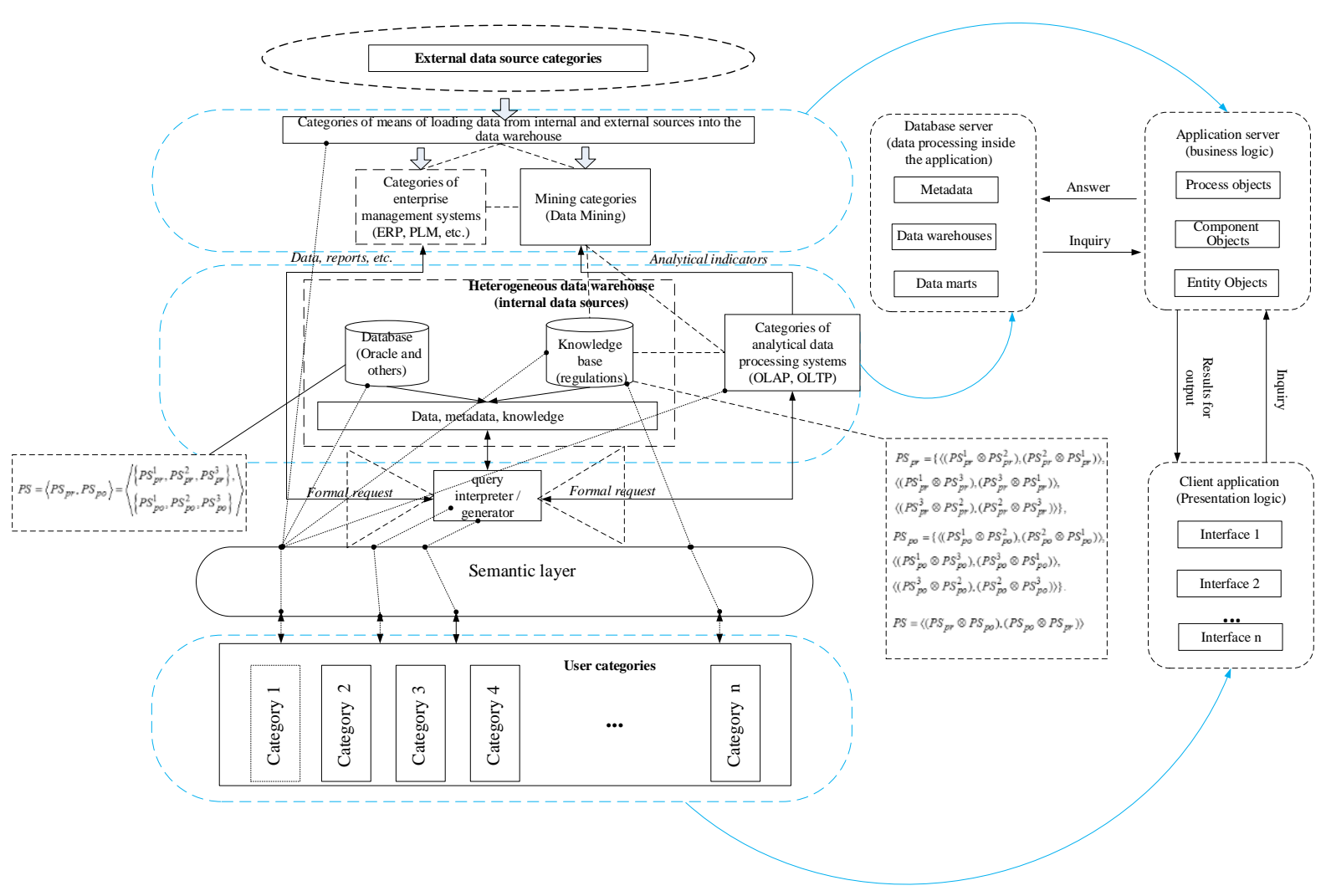

Fig. 3. Generalized data storage scheme SAC 


\section{CONCLUSION}

The result of these studies identified the identifiability and traceability at all levels of SAC on any of the selected chains (information routes) forming the structure of the system. I can conclude that regardless of the information of the routes of formation of SAC by the proposed method, the system properties will be the same. This opens up the possibility of reengineering of the system by choosing a new chain (information routes). Based on the fact that the system of relations is completed before the square of Descartes in each chain of the proposed scheme, there analytical ability to assess completeness. Presents a set-theoretic method for displaying interoperability of software systems in the implementation of the dynamic characteristics of the subject area and the processes of information processing, which deals with the real objects and the projected virtual information objects in the form of a complex multidimensional structure allows to consider the dynamic properties of formal domain models and information processing.

\section{REFERENCES}

[1] Kulikov G.G., Rizvanov K.A., Petrov Yu.E. System approach to the construction of the structure of the organizational-functional model of digital modeling of production processes. Vestnik SUSU. Series: Computer technology, management, electronics. 2018. Vol 2.

[2] Vasilyev V.I. Intellectual control systems. Theory and practice: textbook / B.G. Ilyasov. M .: Radio engineering, 2009. 392p.

[3] Sokolinsky, L.B., Shamakina, A.V.: Methods of resource management in problem-oriented computing environment. Program. Comput. Soft, 2016, 42(1), pp.17-26

[4] Yussupova, Nafissa \& Kovacs, George \& Boyko, Maxim \& Bogdanova, Diana. Models and Methods for Quality Management Based on Artificial Intelligence Applications. Acta Polytechnica Hungarica. 2016 - No. 3. - P. 45-60

[5] Content / [electronic resource] ww.edu.jobsmarket.ru/glossary//3302 / (appeal date: 10/19/16)

[6] Content // www.wikipedia.org/content (access date: 10/19/16)

[7] Feoktistov A., Gorsky S., Sidorov I., Kostromin R., Edelev A., Massel L., Energy Research Application Development Through Convergence of Grid and Cloud Computing //Communications in Computer and Information Science/ Supercomputing, 2019 (in Russian)
[8] Work with personnel reserve // electron. / Url: http://v8.1c.ru/hrm/kadrovij_rezerv/ (circulation date: 08/17/2018)

[9] Vorobev, S., Edelev, A.: Analysis of the importance of critical objects of the gas industry with the method of determining critical elements in networks of technical infrastructures. In: 10th International Conference on Management of Large-Scale System Development (MLSD). IEEE 2017

[10] Bazarova T.Yu., Eremina B.L. Human Resource Management Textbook for High Schools / Ed. Bazarova T.Yu., Eremina B.L. 2nd ed., Moscow: UNITI, 2007. - 560 p.

[11] Baranovskaya, T.P., Vostroknutov, A.E., Berezovsky, V.S. Study of HRM-systems: market analysis, selection and implementation for medium and large businesses // Scientific Journal of KubSAU Scientific Journal of KubSAU. 2016. № 115.

[12] Kovács, G., Yussupova, N., Rizvanov, D. Resource management simulation using multi-agent approach and semantic constraints. An International Journal for Engineering and Information Sciences, 12 (1), 2017, pp. $45-58$

[13] Karnov A.A., Zelenov S.V. Stochastic methods for analysis of complex hardware-software systems // Труды ИСП РАН. 2017. №4. URL:

[14] ISO/IEC/IEEE 15288:2015 System and software engineering System life cycle processes

[15] Гольдблатт Р. Топосы. Категорный анализ логики. М.: Мир, 1983. $-486 \mathrm{c}$

[16] Voropaj N.I., Massel' L.V., Slavin G.B. Setting up the system for monitoring the power engineering facilities in Russia using new information technologies // V. 4, № 1, P. 119-137 (2018) (in Russian)

[17] ISO / IEC 19505: 2012. Information Technology. Unified Object Modeling Group Modeling Language (OMG UML).

[18] Kutluyarov R.V., Lyubopytovab V.S., Bagmanov V.Kh. Sultanov A. $\mathrm{Kh}$. Influence of the linear mode coupling on the nonlinear impairments in few-mode fibers // Procedia Engineering. - 2017 P. 148-154 (in Russian)

[19] Ho K.P., Kahn J.M. Mode coupling and its Impact on spatially multiplexed systems, in: I.P. Kaminow, T. Li and A.E. Willner (Eds.), Optical Fiber Telecommunications VIB: Systems and Networks, Elsevier, San Diego 201319.

[20] Kulikov G.G. Formal presentation of the model for the implementation of system engineering functions based on the principle of the necessary diversity of structural links with the polycubic organization of data conf. comp. Sciences and Inform. tech. (CSIT '2017). Karlsruhe, Germany, 2017. - Ufa: Dialogue, 2017. - V. 1. - P. 92-96. (Article in English. Language) 